\title{
Outcomes Following Neoadjuvant Chemotherapy for Breast Cancer in Women Aged 40 Years and Younger: Impact of Pathologic Nodal Response
}

\author{
Margaret M. Kozak, MDa; Clare E. Jacobson, BAa ; Rie von Eyben, MSª Erqi L. Pollom, MD, MSa; \\ Melinda Telli, $\mathrm{MD}^{\mathrm{b}}$; and Kathleen C. Horst, MDa
}

\begin{abstract}
Purpose: We sought to evaluate whether pathologic nodal response was predictive of outcomes in women aged $\leq 40$ years with breast cancer treated with neoadjuvant chemotherapy (NAC). Methods: A total of 220 patients treated with NAC between 1991 and 2015 were retrospectively reviewed. Pathologic complete response (PCR) was defined as no evidence of residual invasive tumor in the breast and lymph nodes (LNs) (ypTO/Tis ypN0); partial response if there was no tumor in the LNs but residual tumor in the breast (ypT+ypN0) or residual tumor in the LNs (ypT0/Tis ypN+); and limited response if there was residual tumor in both the breast and the LNs (ypT+ ypN+). KaplanMeier and Cox proportional hazards analyses were performed to identify factors predictive for overall survival (OS). Results: A total of 155 patients were included. Following NAC, 39 patients $(25.2 \%)$ achieved pCR, $57(36.8 \%)$ achieved a partial response (either ypT+ ypN0 or ypT0/Tis ypN+), and $59(38.1 \%)$ had a limited response. A total of 22 patients (14.2\%) experienced local failure, 20 (12.9\%) experienced regional failure, and $59(38.1 \%)$ experienced distant failure. Median OS for patients who achieved pCR was not reached, and was significantly worse for patients who had residual disease in the breast and/or LNs $(P<.001)$. No difference in OS was seen among patients who had residual disease in the breast alone versus those who remained LN-positive (97 vs 83 months, respectively; $P=.25$ ). Subset analysis did not reveal differences in OS based on year of treatment or $\mathrm{CN} 1$ disease at the time of initial diagnosis. Conclusions: Women aged $\leq 40$ years who achieved pCR had excellent outcomes; however, those who achieved a pathologic response in the LNs but had residual disease in the breast continued to have outcomes similar to those who remained LN-positive.
\end{abstract}

J Nat/ Compr Canc Netw 2018;16(7):845-850 doi: $10.6004 /$ jnccn.2018.7022

Each year, an estimated 14,000 women aged $\leq 40$ years are diagnosed with breast cancer, which remains the leading cause of cancer-related death in this patient population. ${ }^{1}$ Several studies have shown that breast cancer has more aggressive biological characteristics in women aged $<40$ years compared with older women, ${ }^{2,3}$ including higher likelihood of high-grade, estrogen receptor-negative tumors, and lymph node (LN)-positive disease, ${ }^{4-6}$ However, due to a higher proportion of HER2-positive and triple-negative breast cancer (TNBC) in this group, these women are also more likely

Departments of a Radiation Oncology and ${ }^{b}$ Medicine, Division of Medical Oncology, Stanford Cancer Institute, Stanford, California.

Submitted December 13, 2017; accepted for publication March 2, 2018.

The authors have disclosed that they have no financial interests, arrangements, affiliations, or commercial interests with the manufacturers of any products discussed in this article or their competitors. to have pathologic complete response (pCR) following neoadjuvant chemotherapy (NAC), ${ }^{7-9}$ which has been extensively studied as a surrogate marker for improved prognosis. ${ }^{9-11}$ Clinical trials specifically focusing on this high-risk patient population have not been undertaken, and treatment recommendations are made from extrapolation of available data from trials that predominantly included older, frequently postmenopausal, women. ${ }^{3,12}$

In addition to $\mathrm{pCR}$, complete pathologic nodal response (ypNo) has emerged as a strong predictor of disease-free survival (DFS) and overall survival (OS) in the

Author contributions: Study concept and design: Kozak, Horst. Data acquisition: Kozak, Jacobson. Data analysis and interpretation: Kozak, von Eyben, Horst. Manuscript preparation: All authors. Final approval: Pollom, Telli, Horst.

Correspondence: Kathleen C. Horst, MD, Department of Radiation Oncology, Stanford Cancer Institute, 875 Blake Wilbur Drive, Stanford, CA 94305. Email: kateh@stanford.edu 
neoadjuvant setting, however its prognostic potential has not been investigated in women aged $\leq 40$ years. The current debate centers on whether women who attain either $\mathrm{pCR}$ or $\mathrm{ypNO}$ after NAC should receive adjuvant radiation treatment in the form of regional nodal irradiation or postmastectomy radiation, or whether this can be omitted. In a pooled analysis of NSABP B-18 and B-27,13 attaining pathologic nodal response emerged as a strong predictor of DFS and OS. Another analysis of these 2 studies by Mamounas et $\mathrm{al}^{10}$ showed that younger patients who achieve ypNo have a higher 10-year probability of locoregional recurrence (LRR) compared with women aged $>60$ years; the exact risk for LRR in women aged $<40$ years was not presented. In addition, pathologic nodal status and breast tumor response were predictive of LRR and suggested that, due to low rates of LRR in favorable subgroups, adjuvant regional nodal irradiation and postmastectomy radiation could possibly be omitted. This forms the basis of the rationale for the ongoing NSABP B-51/RTOG 1304 trial (ClinicalTrials.gov identifier: NCT01872975).

Our study evaluated pathologic nodal response in women aged $\leq 40$ years treated with NAC for breast cancer, and hypothesized that it would be associated with DFS and OS in this patient population.

\section{Methods}

We conducted an Institutional Review Board-approved, retrospective, single-institution analysis of women diagnosed with breast cancer at age $\leq 40$ years and treated between 1991 and 2015. A total of 155 patients were identified, 40 and 115 of whom were diagnosed and treated between 1991-2004 and 2005-2015, respectively. All patients received NAC and surgery with or without radiation therapy (RT). Pathologic confirmation of axillary nodal involvement by fine-needle aspiration was not required for inclusion in our analysis. Patients were excluded if surgical or RT records were not available at the time of data analysis, if they had metastatic disease at diagnosis, or if they received chemotherapy for other cancer diagnoses. pCR was defined as no evidence of residual invasive tumor in the breast and sampled axillary LNs (ypTO/Tis ypNo). All other patients were considered to have partial response if there was no tumor in the LNs but residual tumor in the breast (ypT+ ypNo) or no tumor in the breast but residual tumor in the LNs (ypTO/Tis ypN+). Those who had residual tumor in both the breast and $\mathrm{LNs}$ were classified as having a limited response (ypT+ypN+). Residual cancer burden was not routinely assessed in all pathology samples, and therefore was not included in this analysis.

All patients included in this study had detailed clinical and pathologic information available for review, such as clinical stage at diagnosis, hormone receptor (HR) status, HER2 status, Ki67 percentage, BRCA1/2 mutation status, type of NAC, use of HER2-targeted therapy and hormonal therapy, size of residual disease, $\mathrm{LN}$ involvement, presence of lymphovascular invasion, and achievement of $\mathrm{pCR}$. Local (LF), regional (RF), and distant (DF) failure were recorded for each patient.

All statistical analysis was conducted using SAS version 9.4 (SAS Institute Inc.). Competing risks analysis was performed using the Fine-Gray method to analyze cumulative incidence of LF, RF, and DF for each site; hazard ratios were reported with a 95\% CI, and OS was calculated using Kaplan-Meier and logrank tests. Multivariable analysis was performed with Cox proportional hazards regression using select prognostic parameters identified as significant on competing risks analysis. Statistical significance was determined as a 2 -sided $P$ value $<.05$.

\section{Results}

A total of 155 patients were included in this analysis. Median age was 36 years (range, $20-40$ years), and median follow-up time was 52 months (range, 6-293 months). The median OS for the entire cohort was 122 months. Table 1 details the clinical characteristics. In brief, 97 patients $(62.5 \%$ of the entire cohort) had genetic test results available for BRCA, of which $25.8 \%(n=25)$ carried BRCA1 mutations and $7.2 \%(n=7)$ carried BRCA2 mutations. Initial clinical stage at diagnosis was stage I in $4 \%$, stage II in $45 \%$, and stage III in $46 \%$. All patients received NAC followed by mastectomy $(n=108 ; 69.7 \%)$ or lumpectomy $(\mathrm{n}=47 ; 30.3 \%)$. A total of $132(85.2 \%)$ patients received adjuvant RT; 42 of 47 (89.4\%) after lumpectomy (5 refused) and 90 of $108(83.3 \%)$ after mastectomy.

After NAC, 39 patients (25.2\%) achieved pCR (ypTO/Tis ypNo), 52 (33.5\%) achieved ypT+ ypNo, and $5(3.2 \%)$ achieved ypTO/Tis ypN+. The remain- 
Pathologic Nodal Response and Outcomes

\begin{tabular}{|c|c|}
\hline Characteristic & $\mathrm{n}(\%)$ \\
\hline Median age at diagnosis (range), $y$ & $36(20-40)$ \\
\hline \multicolumn{2}{|l|}{ BRCA1/2-positive } \\
\hline Yes & 32 (20.6) \\
\hline No & $65(41.9)$ \\
\hline Unknown & $58(37.4)$ \\
\hline \multicolumn{2}{|l|}{ HR status } \\
\hline Positive & $79(51.0)$ \\
\hline Negative & $74(47.7)$ \\
\hline Unknown & $2(1.3)$ \\
\hline \multicolumn{2}{|l|}{ HER2 receptor status } \\
\hline Positive & $38(24.5)$ \\
\hline Negative/Unknown & $117(75.5)$ \\
\hline \multicolumn{2}{|l|}{ Clinical stage } \\
\hline 1 & $6(3.9)$ \\
\hline II & $70(45.2)$ \\
\hline III & $71(45.8)$ \\
\hline Unknown & $8(5.2)$ \\
\hline \multicolumn{2}{|l|}{ Mastectomy } \\
\hline Yes & $108(69.7)$ \\
\hline No & $47(30.3)$ \\
\hline \multicolumn{2}{|l|}{ Type of axillary surgery } \\
\hline SLNB & $82(52.9)$ \\
\hline ALND & $73(47.1)$ \\
\hline \multicolumn{2}{|l|}{ Axillary lymph nodes removed, $\mathrm{n}$} \\
\hline $1-9$ & $95(61.3)$ \\
\hline $10-20$ & $47(30.3)$ \\
\hline$>20$ & $13(8.4)$ \\
\hline \multicolumn{2}{|l|}{ Pathologic response } \\
\hline урт0/Tis ypNO & $39(25.2)$ \\
\hline ypT+ ypNO & $52(33.5)$ \\
\hline урт0/Tis ypN+ & $5(3.2)$ \\
\hline ypT+ ypN+ & $59(38.1)$ \\
\hline \multicolumn{2}{|l|}{ Adjuvant RT } \\
\hline Yes & $132(85.2)$ \\
\hline No & $23(14.8)$ \\
\hline \multicolumn{2}{|l|}{ Breast cancer subtype $^{a}$} \\
\hline HR-positive/HER2-negative & $60(38.7)$ \\
\hline HR-positive/HER2-positive & $21(13.5)$ \\
\hline HR-negative/HER2-positive & $17(11.0)$ \\
\hline Triple-negative & $55(35.5)$ \\
\hline
\end{tabular}

Abbreviations: ALNB, axillary lymph node biopsy; HR, hormone receptor; RT, radiation therapy; SLNB, sentinel lymph node biopsy. aSubtype was not available for 2 patients.

ing 59 patients $(38.1 \%)$ were found to have residual disease in both the breast and the $\mathrm{LNs}(y p \mathrm{~T}+\mathrm{ypN}+$ ) at the time of final pathologic review. Because only 5 patients achieved ypTO/Tis ypN+, the cohort was subsequently grouped into 3 subsets: patients who attained $\mathrm{pCR}(\mathrm{n}=39)$ and those who did not attain $\mathrm{pCR}$ and were either LN-negative (ypT+ ypNo; $\mathrm{n}=52$ ) or LN-positive (ypN+; $\mathrm{n}=64)$.

A total of 22 patients (14.2\%) experienced LF, 20 (12.9\%) experienced RF, and 59 (38.1\%) experienced DF. LRR occurred among 22 of 108 patients $(20.4 \%)$ who underwent mastectomy and 8 of 47
(17.0\%) who underwent lumpectomy, and DF occurred in 46 of 108 of patients $(43.6 \%)$ who underwent mastectomy and 13 of $47(27.6 \%)$ who underwent lumpectomy. No local or regional recurrences occurred in patients who achieved pCR. A total of 3 patients $(7.7 \%)$ with pCR developed distant metastases, 2 of whom were still alive with disease at time of writing, and 1 who died 96 months after the initial diagnosis. However, patients with residual disease in either the breast or LNs had a higher incidence of LF, RF, and DF than those who achieved pCR (Figure 1), with approximately 50\% experiencing DF at 10 years $(P<.001$; Table 2$)$. Of those who achieved a pCR in the LNs, patients who still had residual disease in the breast did not have better outcomes compared with those who remained LN-positive. Cumulative incidence of LF, RF, and $\mathrm{DF}$ at 10 years by response to NAC is depicted in Table 2, with corresponding cumulative incidence curves in Figure 1. Median OS for patients with pCR was not reached $(P<.001)$. No significant difference in $\mathrm{OS}$ was seen between ypT+ ypNo versus ypN+ patients, with median OS of 97 versus 83 months, respectively $(P=.25)$ (Figure $2 \mathrm{~A}$ ).

To evaluate whether modern systemic therapy affected OS, we stratified patients into 2 groups based on treatment date and chose a cutoff of 2005 , because this was when the use of trastuzumab for HER2-positive breast cancer had become standard of care. On Kaplan-Meier analysis, no difference in OS was seen among patients treated between 19912004 and 2005-2015 (median OS, 122 months vs not reached, respectively; $P=.88$ ). We next analyzed patients treated between 2005-2015 ( $n=116)$ separately to evaluate outcomes in a patient population with routine HER2 testing and regular use of trastuzumab. Patients with ypT + ypNo and ypN + disease continued to have worse OS compared with those who achieved pCR, with median OS not reached for the pCR group, and 84 versus 77 months, respectively, for patients with ypT + ypNO and ypN+ disease $(P<.001$; Figure $2 \mathrm{~B})$. We also evaluated outcomes of patients with an initial diagnosis of clinical T1-3N1 breast cancer $(\mathrm{n}=115)$ who achieved $\mathrm{pCR}$ in the LNs (ypTO/Tis ypNO and ypT+ ypNO) following NAC, because this group is included in the ongoing NSABP B-51 trial. We found that outcomes of patients who achieved pCR were excellent, with median $\mathrm{OS}$ not reached $(P=.03$; Figure $2 \mathrm{C})$; how- 
A

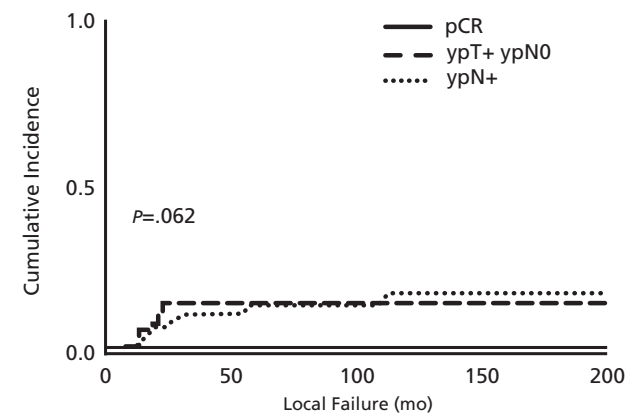

B

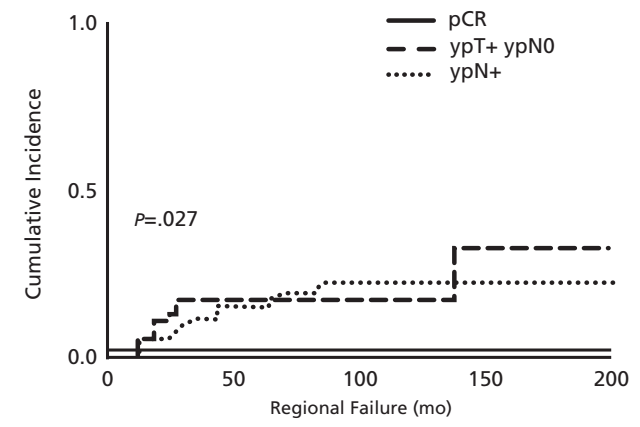

C

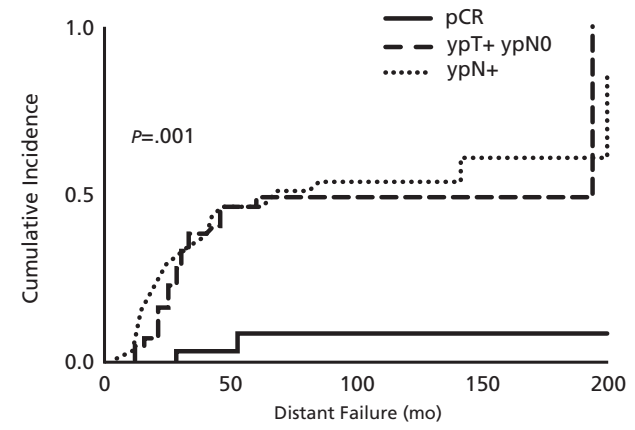

Figure 1. Cumulative incidence of (A) local, (B) regional, and (C) distant failure by pathologic nodal response.

Abbreviations: $\mathrm{pCR}$, pathologic complete response; $y \mathrm{pN}+$, residual tumor in the lymph nodes; ypT+ ypN0, no tumor in the lymph nodes but residual tumor in the breast.

ever, patients with ypT+ ypNo disease did not have improved OS compared with those who remained ypN+ after NAC, with a median OS of 84 versus 107 months, respectively. On multivariable analysis, mastectomy $(P=.009)$, adjuvant RT $(P=.045)$, achievement of pCR $(P<.001)$, and total LNs sampled $(P=.011)$ were all predictive of OS (Table 3$)$.

Lastly, we investigated the impact of breast cancer subtype on outcomes. Patients were divided into 4 groups: HR-positive/HER2-negative $(n=60$; 39.2\%), HR-positive/HER2-positive ( $n=21 ; 13.7 \%)$, HR-negative/HER2-positive $(\mathrm{n}=17 ; 11.1 \%)$, and TNBC ( $\mathrm{n}=55 ; 35.9 \%)$; subtype was not available for 2 patients. pCR by breast cancer subtype was $18.3 \%$

\begin{tabular}{|lcccc|}
\hline Table 2. Ten-Year Cumulative Incidence of Local, \\
Regional, and Distant Failure
\end{tabular}

Abbreviations: $\mathrm{LN}$, lymph node; $\mathrm{pCR}$, pathologic complete response; $\mathrm{ypN}+$, residual tumor in the LNs; ypT+ ypNO, residual tumor in the breast but no tumor in the LNs.

for HR-positive/HER2-negative, 23.8\% for HRpositive/HER2-positive, $35.3 \%$ for HR-negative/ HER2-positive, and 31\% for TNBC. Median OS was not reached for the HR-positive/HER2-positive and TNBC cohorts, was 170 months in the HR-positive/ HER2-negative cohort, and was 96 months in the HR-negative/HER2-positive cohort $(P=.03)$. OS by subtype and $\mathrm{pCR}$ is shown in supplemental eFigure 1 (available with this article at JNCCN.org).

\section{Discussion}

To our knowledge, this is the first study evaluating outcomes by pathologic nodal response in women aged $\leq 40$ years treated with NAC. We focused on young women treated with NAC because this population is infrequently studied on its own, yet has worse survival compared with older women despite higher rates of $\mathrm{pCR}$. In addition, although the secondary analysis of NSABP B-18 and B- $27^{10}$ has been used to guide risk assessment following NAC, it does not include a comprehensive analysis of recurrence risk in women aged $\leq 40$ years. Our report presents LF, RF, and DF rates in this high-risk breast cancer population, making this analysis unique with respect to the published literature.

Our analysis reveals that women aged $\leq 40$ years with residual disease in the breast or LNs have increased LRR rates compared with those who achieve pCR in both the breast and LNs. Perhaps the most compelling finding of our analysis is that women with residual disease in the breast, despite having a pCR in the LNs, had similar LRR rates as those with residual nodal disease. This important finding suggests that omitting RT in patients with any residual disease after NAC, whether in the breast alone or in the breast and LNs, should be considered with caution. The LRR rates remained between $15 \%$ and $22 \%$ in patients with yp $\mathrm{T}+\mathrm{ypNO}$ and ypN+ disease, even though most patients in our study received 
Pathologic Nodal Response and Outcomes

A

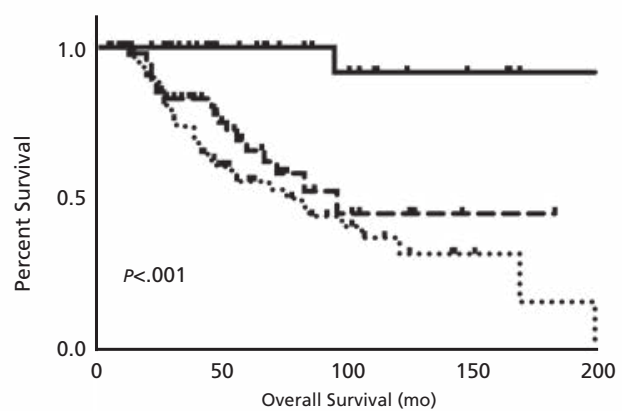

\begin{tabular}{lllrll} 
Number at risk & & & & & \\
- pCR & 39 & 22 & 10 & 5 & 1 \\
\hline$-1-$ ypT+ ypNo & 52 & 29 & 7 & 2 & 0 \\
$\ldots \ldots$... ypN+ & 64 & 29 & 13 & 3 & 1
\end{tabular}

B

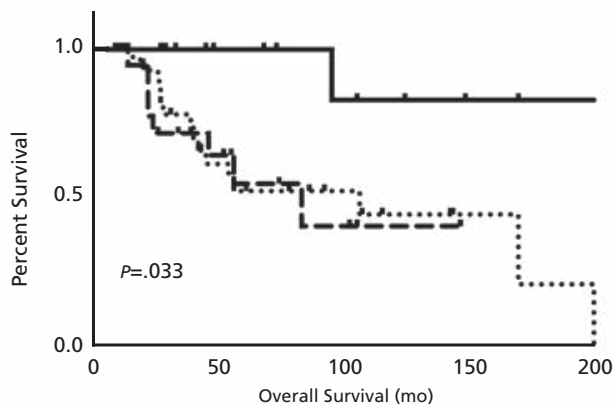

Number at risk

\begin{tabular}{|c|c|c|c|c|}
\hline$\longrightarrow \mathrm{pCR}$ & 17 & 9 & 6 & 3 \\
\hline -1- урT+ урNo & 20 & 10 & 4 & 0 \\
\hline ...... ypN+ & 30 & 15 & 8 & 3 \\
\hline
\end{tabular}

C

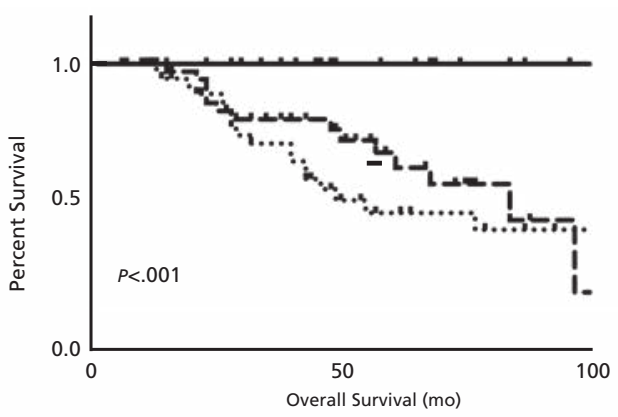

Number at risk

- $\mathrm{pCR} \quad 31$

....... ypN+ 43

15
22

15

4
2

6

Figure 2. Overall survival for $(A)$ entire cohort, $(B)$ patients treated from 2005-2015, and (C) patients with initial diagnosis of clinical T13N1 breast cancer.

Abbreviations: $\mathrm{pCR}$, pathologic complete response; $\mathrm{ypN}+$, residual tumor in the lymph nodes; ypT+ ypN0, residual tumor in the breast but no tumor in the lymph nodes.

whole-breast/chest wall RT with nodal irradiation. Patients with residual disease also had high rates of $\mathrm{DF}$ and poor OS, and no difference in DF or OS was seen between patients with ypT+ ypNO and those with ypT+ypN + disease. These results suggest that any residual disease following NAC, whether in the

\begin{tabular}{|lccc|}
\hline \multicolumn{4}{|l|}{$\begin{array}{l}\text { Table 3. Multivariable Analysis of Prognostic } \\
\text { Variables for Overall Survival }\end{array}$} \\
\hline Variable & $\boldsymbol{P}$ Value & Hazard Ratio & $95 \% \mathrm{Cl}$ \\
\hline Mastectomy & .009 & 0.392 & $0.195-0.789$ \\
\hline Adjuvant RT & .045 & 0.336 & $0.116-0.974$ \\
\hline pCR & $<.001$ & 0.075 & $0.021-0.267$ \\
\hline Total LNs sampled & .011 & 0.950 & $0.913-0.988$ \\
\hline
\end{tabular}

Abbreviations: LNs, lymph nodes; pCR, pathologic complete response; $\mathrm{RT}$, radiation therapy.

breast alone or in the breast and the LNs, is a predictor of poor prognosis in this patient population. In contrast, no patient who achieved pCR following NAC experienced LRR, and the incidence of DF was low. These results corroborate other published series showing $\mathrm{pCR}$ to be the strongest predictor of outcome after NAC. ${ }^{10,14}$ Whether patients who achieve a pCR will have low rates of LRR without postmastectomy or nodal irradiation is an area of ongoing investigation.

A large percentage of our cohort (38.1\%) experienced DF, driven in large part by women who did not achieve pCR following NAC. This high rate of $\mathrm{DF}$ is also likely due to most of these women ( $\approx 90 \%)$ having stage II-III disease at diagnosis, making our patient population particularly high risk. A retrospective analysis of 170 women aged $\leq 40$ years treated with NAC also observed a high rate of recurrence $(29.4 \%)$ and mortality (22.9\%). ${ }^{15}$ It is likely that these women would benefit from adjuvant treatment to improve outcomes, and several trials have evaluated escalation of adjuvant therapy. ${ }^{16-18}$ The recently published CREATE-X trial ${ }^{19}$ evaluated 910 patients with HER2-negative breast cancer who had residual invasive disease or LN metastases after NAC. Women were randomized to either adjuvant capecitabine or observation. At 5 years of follow-up, the addition of adjuvant capecitabine significantly prolonged DFS and OS. Other efforts to improve outcomes center around increasing rates of $\mathrm{pCR}$ through the escalation of NAC regimens. ${ }^{20,21}$ In the 5-year follow-up of the NeoSphere trial, ${ }^{22}$ the addition of pertuzumab to trastuzumab + docetaxel improved long-term outcomes in women with HER2-positive breast cancer. This led to the accelerated FDA approval of pertuzumab in the neoadjuvant setting. ${ }^{23}$

Our analysis shows that young women with breast cancer who achieve a pCR in both the breast and the LNs experience improved outcomes, whereas those who have residual disease in the breast, de- 
spite having a pCR in the LNs, do not have better outcomes compared with those who remain LNpositive after NAC. The strengths of this study include a detailed assessment of pathologic response and patterns of failure, as well as stratification of patients based on the time frame during which they received treatment (1991-2004 vs 2005-2015), clinical stage at diagnosis, and breast cancer subtype. Our analysis differs from previously reported studies because it assesses LRR, as well as DF and OS for all patients included in the analysis. Limitations of our study include its retrospective nature and relatively small sample size. As a single-institution study, our results may not be generalizable to the population at large, but are hypothesis-generating nonetheless.

\section{Conclusions}

Our analysis demonstrates that outcomes in young women aged $\leq 40$ years receiving NAC vary by pathologic response in the breast and the LNs. Young women who achieve pCR have excellent outcomes; however, those who achieve pCR in the LNs but have residual disease in the breast continue to have outcomes similar to those who remain LN-positive. Our data suggest that de-escalation of care in this patient population should be approached with caution until the results of NSABP B-51 are available.

\section{References}

1. Siegel RL, Miller KD, Jemal A. Cancer statistics, 2016. CA Cancer J Clin 2016;66:7-30.

2. Villarreal-Garza C, Mohar A, Bargallo-Rocha JE, et al. Molecular subtypes and prognosis in young Mexican women with breast cancer. Clin Breast Cancer 2017;17:e95-102.

3. Narod SA. Breast cancer in young women. Nat Rev Clin Oncol 2012;9:460470 .

4. Collins LC, Marotti JD, Gelber S, et al. Pathologic features and molecular phenotype by patient age in a large cohort of young women with breast cancer. Breast Cancer Res Treat 2012;131:1061-1066.

5. Gnerlich JL, Deshpande AD, Jeffe DB, et al. Elevated breast cancer mortality in women younger than age 40 years compared with older women is attributed to poorer survival in early-stage disease. J Am Coll Surg 2009;208:341347.

6. Keegan TH, DeRouen MC, Press DJ, et al. Occurrence of breast cancer subtypes in adolescent and young adult women. Breast Cancer Res 2012;14:R55.

7. Azim HA, Michiels S, Bedard PL, et al. Elucidating prognosis and biology of breast cancer arising in young women using gene expression profiling. Clin Cancer Res Res 2012;18:1341-1351.

8. Anders CK, Carey LA. Biology, metastatic patterns, and treatment of patients with triple-negative breast cancer. Clin Breast Cancer 2009;9(Suppl 2):S73-81.

9. von Minckwitz G, Untch M, Blohmer JU, et al. Definition and impact of pathologic complete response on prognosis after neoadjuvant chemotherapy in various intrinsic breast cancer subtypes. J Clin Oncol 2012;30:1796-1804.

10. Mamounas EP, Anderson SJ, Dignam JJ, et al. Predictors of locoregional recurrence after neoadjuvant chemotherapy: results from combined analysis of National Surgical Adjuvant Breast and Bowel Project B-18 and B-27. J Clin Oncol 2012;30:3960-3966.

11. Cortazar P, Zhang L, Untch M, et al. Pathological complete response and long-term clinical benefit in breast cancer: the CTNeoBC pooled analysis. Lancet 2014;384:164-172.

12. Shannon C, Smith IE. Breast cancer in adolescents and young women. Eur J Cancer 2014;39:2632-2642.

13. Rastogi $\mathrm{P}$, Anderson SJ, Bear HD, et al. Preoperative chemotherapy: update of National Surgical Adjuvant Breast and Bowel Project protocols B-18 and B-27. J Clin Oncol 2008;26:778-785.

14. Buchholz TA, Tucker SL, Masullo L, et al. Predictors of local-regional recurrence after neoadjuvant chemotherapy and mastectomy without radiation. J Clin Oncol 2002;20:17-23.

15. Spring L, Greenup R, Niemierko A, et al. Pathologic complete response after neoadjuvant chemotherapy and long-term outcomes among young women with breast cancer. J Natl Compr Cancer Netw 2017;15:1216-1223.

16. Joensuu H, Kellokumpu-Lehtinen PL, Huovinen R, et al. Adjuvant capecitabine, docetaxel, cyclophosphamide, and epirubicin for early breast cancer: final analysis of the randomized FinXX trial. J Clin Oncol 2012;30:11-18.

17. Joensuu H, Kellokumpu-Lehtinen PL, Huovinen R, et al. Adjuvant capecitabine in combination with docetaxel, epirubicin, and cyclophosphamide for early breast cancer: the randomized clinical FinXX trial. JAMA Oncol 2017;3:793-800.

18. Martín M, Ruiz Simón A, Ruiz Borrego M, et al. Epirubicin plus cyclophosphamide followed by docetaxel versus epirubicin plus docetaxel followed by capecitabine as adjuvant therapy for node-positive early breast cancer: results from the GEICAM/2003-10 study. J Clin Oncol 2012;33:3788-3795.

19. Masuda N, Lee SJ, Ohtani S, et al. Adjuvant capecitabine for breast cancer after preoperative chemotherapy. N Engl J Med 2017;376:2147-2159.

20. Bear HD, Tang G, Rastogi $P$, et al. Neoadjuvant plus adjuvant bevacizumab in early breast cancer (NSABP B-40 [NRG Oncology]): secondary outcomes of a phase 3, randomised controlled trial. Lancet Oncol 2015;16:1037-1048.

21. de Azambuja E, Holmes AP, Piccart-Gebhart M, et al. Lapatinib with trastuzumab for HER2-positive early breast cancer (NeoALTTO): survival outcomes of a randomised, open-label, multicentre, phase 3 trial and their association with pathological complete response. Lancet Oncol 2014;15:1137-1146

22. Gianni L, Pienkowski T, Im YH, et al. 5-year analysis of neoadjuvant pertuzumab and trastuzumab in patients with locally advanced, inflammatory, or early-stage HER2-positive breast cancer (NeoSphere): a multicentre, openlabel, phase 2 randomised trial. Lancet Oncol 2016;17:791-800.

23. Amiri-Kordestani L, Wedam S, Zhang L, et al. First FDA approval of neoadjuvant therapy for breast cancer: pertuzumab for the treatment of patients with HER2-positive breast cancer. Clin Cancer Res 2014;20:5359-5364. 